Supplementary Information for:

\title{
Removal of heavy metal ions from water by magnetic cellulose-based beads with embedded chemically modified magnetite nanoparticles and activated carbon
}

\author{
Xiaogang Luo*, Xiaojuan Lei, Ning Cai, Xiuping Xie, Yanan Xue, Faquan $\mathrm{Yu}^{*}$
}

Key Laboratory for Green Chemical Process of Ministry of Education; Hubei Key

Laboratory for Novel Reactor and Green Chemistry Technology; School of Chemical

Engineering and Pharmacy, Wuhan Institute of Technology, Wuhan 430073, China

Total pages: 2 excluding cover page

Total Tables: 1

Total Figures: 2

\footnotetext{
* Corresponding author: Xiaogang Luo, Professor, Ph.D.

School of Chemical Engineering and Pharmacy, Wuhan Institute of Technology, Wuhan 430073, Hubei, China

Tel.: +86-139-86270668;

Email:xgluo@wit.edu.cn; xgluo0310@hotmail.com (X. Luo)

Corresponding author: Faquan Yu, Professor, Ph.D.

School of Chemical Engineering and Pharmacy, Wuhan Institute of Technology, Xiongchu Ave, Wuhan 430073, Hubei, China

Tel: (+86-27) 8719-4980; Fax: (+86-27) 8719-4465

E-mail: fyu@wit.edu.cn; fyuwucn@gmail.com
} 
Table S1 Physical properties of native cellulose beads and MCB

\begin{tabular}{ccccc|cc}
\hline \multicolumn{5}{c|}{ Drainage method } & \multicolumn{2}{c}{ BET method } \\
\hline Sample & $\begin{array}{c}\text { Water } \\
\text { content } \omega \\
(\%)\end{array}$ & $\begin{array}{c}\text { Wet density } \\
\rho_{w} \\
\left(\mathrm{~g} \mathrm{ml}^{-1}\right)\end{array}$ & $\begin{array}{c}\text { Pore } \\
\text { volume } \\
V_{\mathrm{p}}\left(\mathrm{ml} \mathrm{g}^{-1}\right)\end{array}$ & $\begin{array}{c}\text { Porosity } P_{\mathrm{r}} \\
(\%)\end{array}$ & $\begin{array}{c}\text { BET } \\
\text { surface } \\
\text { area } S \\
\left(\mathrm{~m}^{2} / \mathrm{g}\right)\end{array}$ & $\begin{array}{c}\text { Average } \\
\text { Pore } \\
\text { diameter } \\
D(\mathrm{~nm})\end{array}$ \\
\hline $\begin{array}{c}\text { native } \\
\text { cellulose } \\
\text { beads }\end{array}$ & $91.95 \pm 0.02$ & $1.05 \pm 0.14$ & $\begin{array}{c}9.22 \pm \\
0.16\end{array}$ & $\begin{array}{c}92.60 \pm \\
0.10\end{array}$ & 88.58 & 864 \\
MCB & $91.20 \pm 0.04$ & $1.10 \pm 0.10$ & $\begin{array}{c}9.99 \pm \\
0.26\end{array}$ & $\begin{array}{c}94.40 \pm \\
0.20\end{array}$ & 90.05 & 220 \\
\hline
\end{tabular}
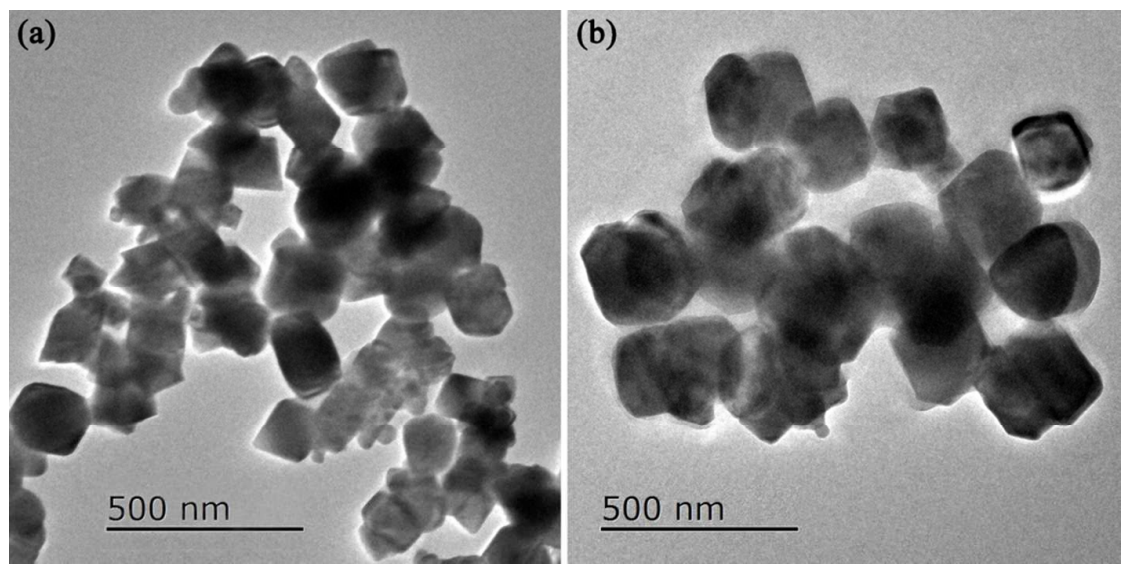

Figure $\mathrm{S} 1$. TEM image of $\mathrm{Fe}_{3} \mathrm{O}_{4}$ nanoparticles (a) and modified $\mathrm{Fe}_{3} \mathrm{O}_{4}$ nanoparticles
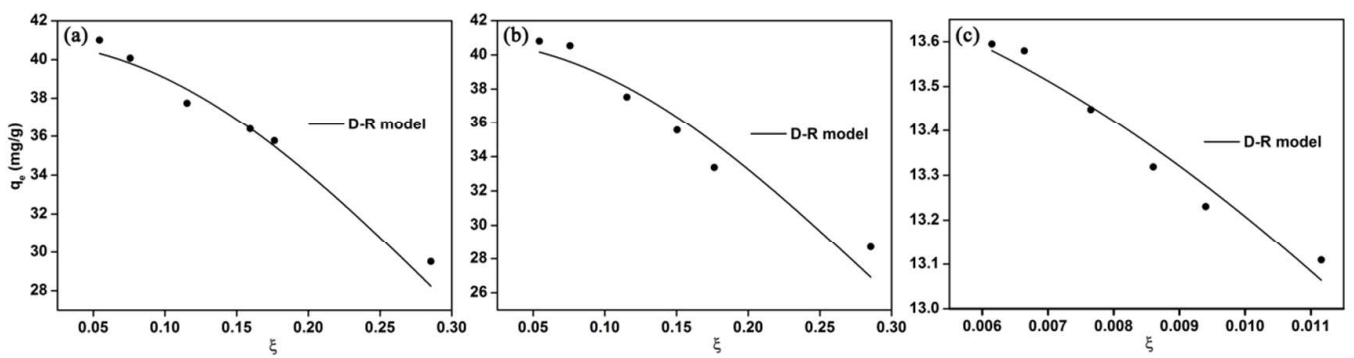

Figure S2. D-R isotherm of $\mathrm{Cu}^{2+}(\mathrm{a}), \mathrm{Pb}^{2+}(\mathrm{b})$ and $\mathrm{Zn}^{2+}$ (c) onto $\mathrm{MCB}$ at $298 \mathrm{~K}$ 